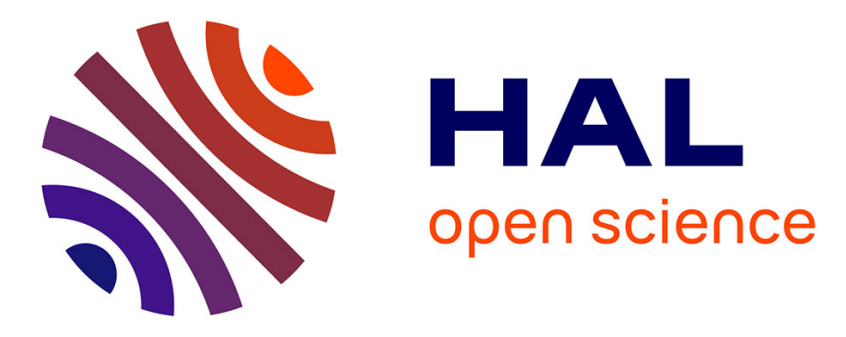

\title{
On Signal Space Diversity for non binary coded modulation schemes
}

\author{
Ahmed Abdmouleh, Emmanuel Boutillon, Laura Conde-Canencia, Charbel \\ Abdel Nour, Catherine Douillard
}

\section{- To cite this version:}

Ahmed Abdmouleh, Emmanuel Boutillon, Laura Conde-Canencia, Charbel Abdel Nour, Catherine Douillard. On Signal Space Diversity for non binary coded modulation schemes. 23rd International Conference on Telecommunications, May 2016, Thessaloniki, Greece. pp.1 - 5, 10.1109/ICT.2016.7500494 . hal-01426871

\section{HAL Id: hal-01426871 \\ https://hal.science/hal-01426871}

Submitted on 5 Jan 2017

HAL is a multi-disciplinary open access archive for the deposit and dissemination of scientific research documents, whether they are published or not. The documents may come from teaching and research institutions in France or abroad, or from public or private research centers.
L'archive ouverte pluridisciplinaire HAL, est destinée au dépôt et à la diffusion de documents scientifiques de niveau recherche, publiés ou non, émanant des établissements d'enseignement et de recherche français ou étrangers, des laboratoires publics ou privés. 


\title{
On Signal Space Diversity for Non Binary Coded Modulation Schemes
}

\author{
Ahmed Abdmouleh\#, Emmanuel Boutillon \#, Laura Conde-Canencia \#, Charbel Abdel Nour* and \\ Catherine Douillard* \\ \# Université de Bretagne Sud, Lab-STICC (UMR 6582), 56321 Lorient, France \\ *Institut Mines-Telecom; Telecom Bretagne; Lab-STICC (UMR 6582), Technopole Brest-Iroise, 29238 Brest, France \\ \# ahmed.abdmouleh, emmanuel.boutillon, laura.conde-canencia@univ-ubs.fr \\ * catherine.douillard, charbel.abdelnour@telecom-bretagne.eu
}

\begin{abstract}
In this paper, we study high-spectral efficiency and high-order diversity communications for the next generation broadcasting systems. In this context, rotating a Quadrature Amplitude Modulation constellation with a Signal Space Diversity (SSD) technique is known to improve the decoding performance of the communication system over the Rayleigh fading channel. In this work, we consider the SSD technique applied to coded modulation schemes with the novelty of selecting the rotation angle from the analysis of the mutual information variations. In this context, it is known that coding modulation outperforms bit-interleaved coded modulation, but we show that coded modulation has also an extra advantage: the optimal rotation angle is only marginally impacted by the signal to noise ratio and the erasure rate of the fast Rayleigh fading channel. This opens the door to a robust coded modulation scheme particularly suited for broadcasting applications. Simulation results confirm the theoretical study with a gain of up to 1.2 dB when comparing to the DVB-T2 standard.
\end{abstract}

Index Terms-Coded Modulation ; Bit Interleaved Coded Modulation ; Rotated Constellation ; Signal Space Diversity ; Mutual information

\section{INTRODUCTION}

Next generation broadcast systems such as DVB-T2 [1] and DVB-NGH [2] must guarantee robust high data rate transmissions in severe channels. A key aspect in this context is the increase of the diversity order while keeping high spectral efficiency. Currently, the Signal Space Diversity (SSD) [3] principle associated to Bit-Interleaved Coded Modulation (BICM) represents the reference in coded modulation over fading channels. BICM was first introduced by Zehavi in [4] and later formalised by Caire et al. in [5]. Its principle is to serially concatenate a Forward Error Correction (FEC) code and a bit-interleaver prior to the mapping and modulation step. Then, since the channel encoder and the modulator are separated by a bit-level interleaver, they can be chosen independently allowing for a simple and flexible design.

The SSD technique consists of rotating the constellation in the signal space and interleaving one of the In-phase (I) or Quadrature (Q) signal components with respect to the other. The interleaving makes the I and Q components to be sent in different symbol periods, each being affected by a different fading coefficient. This doubles the diversity order of the BICM scheme. These so-called Rotated Contellations (RC) were adopted in the DVB-T2 [1] and DVB-NGH [2] standards.
In this paper, we investigate the association of $q$-ary codes to high-order modulations, where the cardinality of the code equals the modulation order. This kind of scheme is called Coded Modulation (CM). We consider the optimization of the SSD technique through the analysis of the mutual information of the channel as a function of the rotation angle. This approach is applied to both BICM and $\mathrm{CM}$ schemes to show the interest and advantages of the CM solution. Note that for the BICM we consider a binary FEC code whereas for the CM scheme we adopt a Non-Binary (NB) code defined over a Galois Field GF $(q)$, which allows to directly map a coded symbol to a constellation point. In [6] and [7] the authors provide results on the association of NB-LDPC and high-order modulations with rotated constellations to show the interest of the $\mathrm{CM}$ scheme. A gain of about $0.15 \mathrm{~dB}$ is introduced with the SSD in a NB-LDPC coded modulation scheme [6].

The paper is organised as follows: Section II presents the principle of the SSD technique and its application to the DVB-T2 standard. Then, a theoretical study of the mutual information is presented in Section III. Section IV describes how the analysis of the mutual information can optimize the SSD technique for coded modulations. Simulation results and discussions are presented in Section V. Finally, Section VI draws conclusions.

\section{Signal Space Diversity}

When a constellation symbol $x=\left(x_{I}, x_{Q}\right)$ is directly transmitted in the Rayleigh fading channel, both the I $\left(x_{I}\right)$ and $\mathrm{Q}\left(x_{Q}\right)$ components fade identically. In case of severe fading, an irreversible loss of information on both components can be observed. In order to help the demapper to retrieve the transmitted symbol, it is more efficient to send separately $x_{I}$ and $x_{Q}$ in two different time or frequency slots, so that the fading coefficient $r_{I}$ affecting $x_{I}$ is independent of the fading coefficient $r_{Q}$ affecting $x_{Q}$.

In the SSD scheme adopted in the DVB-T2 standard [8], the encoded symbol is mapped to the constellation to generate a modulated signal $x^{t}=\left(x_{I}^{t}, x_{Q}^{t}\right)$, where $t$ represents the time index. Before transmission, the quadrature component $x_{Q}^{t}$ is delayed by $d$ symbols. In others words, at time $t$, the transmitted symbol $\left(x_{I}^{t}, x_{Q}^{t-d}\right)$ is affected by a multiplicative factor $r^{t}$ and at time $t+d$, the transmitted symbol $\left(x_{I}^{t+d}, x_{Q}^{t}\right)$ is affected by $r^{t+d}$. At 
the receiver side, a delay on the I component allows to reconstruct the received symbol $y^{t}$, with $y_{I}^{t}=r_{I}^{t} x_{I}^{t}+w_{I}$ and $y_{Q}^{t}=r_{Q}^{t} x_{Q}^{t}+w_{Q}$, where $w_{I}$ and $w_{Q}$ represent the additive noise on the I and Q axes, $r_{I}^{t}=r^{t}$ and $r_{Q}^{t}=r^{t+d}$.

Fig. 1 illustrates the effect of the Rayleigh fading channel on a Quadrature Amplitude Modulation (QAM) with 16 points, with and without rotation: the I axis suffers a severe fading $\left(r_{I}=0.2\right)$ while there is no fading on the $\mathrm{Q}$ axis $\left(r_{Q}=1\right)$. In the first case, the reconstructed constellation has points very close one to each other, which increases the error probability. On the contrary, with the rotated constellation, the minimum distance between the points of the reconstructed constellation remains significant.

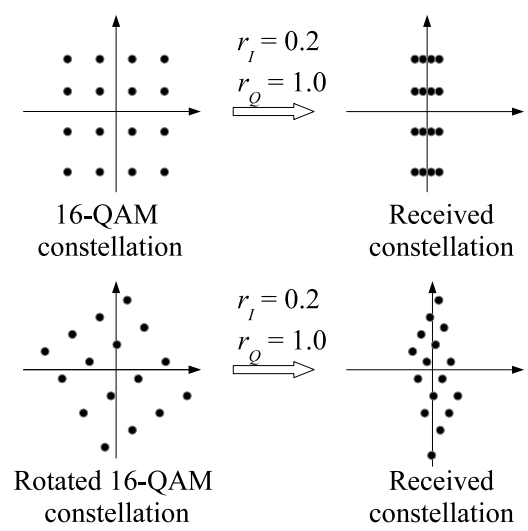

Fig. 1. 16-QAM constellation, $37.2^{\circ}$-rotated 16-QAM constellation before transmission and after reception for fading factors of $r_{I}=1$ on the $I$-axis and $r_{Q}=0.2$ on the $Q$-axis.

\section{A. Choice of the rotation angle $\alpha$ in the DVB-T2 standard}

As the choice of the SSD rotation angle $\alpha$ depends on different factors, different solutions have been considered in the literature. In [3] the choice of $\alpha$ aims to maximize the so-called product distance in order to minimize the pairwise error probability between two different transmitted sequences. Other criteria are considered in [9] like the average Hamming distance between any two adjacent constellation symbols in the 2-D constellation and the incidence on the Gray mapping in the projected constellation points after rotation. Unfortunately, in practice, these criteria are in conflict and their simultaneous application leads to different values of $\alpha$. The angle values adopted in the DVB-T2 standard [1] represent a compromise between the listed criteria. They are given in Table-I for different modulation orders.

\begin{tabular}{cc}
\hline Constellation & Rotation Angle $\alpha$ value (degree) \\
\hline QPSK & $29.0^{\circ}$ \\
16-QAM & $16.8^{\circ}$ \\
64-QAM & $8.6^{\circ}$ \\
256-QAM & $3.6^{\circ}$ \\
\hline
\end{tabular}

VALUES OF THE ROTATION ANGLES IN THE DVB-T2 STANDARD

As already introduced in section I, the novelty of the paper is to select the rotation angle that maximizes the mutual information. The following Section is then dedicated to the theoretical study of the mutual information of the $\mathrm{CM}$ and BICM schemes.

\section{MUtUAL INFORMATION}

\section{A. Notations}

The considered constellation is denoted by $\mathcal{Q}$. It contains $q$ points $x_{k}, k=1 \cdots q$, where $x_{k}$ is defined by its in-phase $x_{I}^{k}$ and quadrature $x_{Q}^{k}$ components, i.e., $x_{k}=\left(x_{I}^{k}, x_{Q}^{k}\right)=x_{I}^{k}+j x_{Q}^{k}$. Assuming perfect Channel State Information (CSI) at the receiver side, the received point $y=y_{I}+j y_{Q}$ after transmission of $x \in \mathcal{Q}$ over the fast flat Rayleigh fading channel with erasures is given by $y=r_{I} e_{I} x_{I}+j e_{Q} r_{Q} x_{Q}+w$ where $w$ is a complex Gaussian noise with variance $\sigma^{2}, r_{I}$ and $r_{Q}$ are independent realizations of a Rayleigh fading with unitary standard deviation and $e_{I}, e_{Q}$ are the erasure coefficients. If we consider the Rayleigh channel without erasure then $e_{I}=e_{Q}=1$, while for the Rayleigh channel with erasures, $P\left(e_{I}=0\right)=P\left(e_{Q}=0\right)=p_{e}$ and $P\left(e_{I}=1\right)=P\left(e_{Q}=1\right)=1-p_{e}$, where $p_{e}$ is the erasure probability. In the sequel, $r$ will denote the couple $\left(r_{I}, r_{Q}\right)$ and we will consider the channel without erasure for the theoretical equations that follow. At the receiver side, $P(x \mid y ; r)$, the probability that $x$ has been transmitted given $y$ and $r$ is defined as:

$$
P(x \mid y ; r)=\frac{P(y \mid x ; r) P(x)}{\sum_{k=1}^{q} P\left(y \mid x_{k} ; r\right) P\left(x_{k}\right)}
$$

with

$$
P(y \mid x ; r)=\frac{1}{\sqrt{2 \pi} \sigma} e^{-\frac{\left(y_{I}-r_{I} x_{I}\right)^{2}+\left(y_{Q}-r_{Q} x_{Q}\right)^{2}}{2 \sigma^{2}}} .
$$

\section{B. Mutual information computation}

Let us define $X$ and $Y$ as the random variables that represent the input and output of the transmission channel. The mutual information $I(X ; Y)$ between $X$ and $Y$ is given by

$$
I(X ; Y)=H(X)-H(X \mid Y) .
$$

The first term in (3) is maximized when all the constellation points have the same uniform probability $1 / q$ of being selected. In that case, $H(X)=$ $-\sum_{k=1}^{q} P\left(x_{k}\right) \log _{2}\left(P\left(x_{k}\right)\right)=m$, where $m=\log _{2}(q)$. The second term in (3) is more complex to compute. We can derive its expression in the case of CM or BICM.

1) Mutual information in the CM scheme: For CM, the $q$-ary coded symbol is directly mapped to a constellation point. The second term in (3) is then expressed as

$$
H(X \mid Y)=-\int_{y \in \mathbb{C}} p(y)\left(\int_{r \in \mathbb{R}^{2}} H(X \mid y ; r) P(r) \mathrm{d} r\right) \mathrm{d} y
$$

where $\mathbb{R}$ is the set of real numbers, $\mathbb{C}$ is the set of complex numbers and $H(X / y ; r)$ is defined as

$$
H(X \mid y ; r)=-\sum_{k=1}^{q} P\left(x_{k} \mid y ; r\right) \log _{2}\left(P\left(x_{k} \mid y ; r\right)\right) .
$$


2) Mutual information in the BICM scheme: Let us consider a binary mapping of the constellation $\mathcal{Q}$ that associates to each point $x_{k}$ a binary codeword $\left(b_{k, 1}, \cdots b_{k, m}\right)$. Let $X_{i}^{0}$ be the set of symbols where the $i^{\text {th }}$ bit $b_{i}$ is equal to 0 and $X_{i}^{1}$ be the set of symbols where the $i^{t h}$ bit $b_{i}$ is equal to 1 . Thus,

$$
P\left(b_{i}=s \mid y ; r\right)=\sum_{x \in X_{i}^{s}} P(x \mid y ; r), s=0,1 .
$$

Assuming ideal bit interleaving, which makes the considered channel equivalent to $m$ binary channels ([5] eq. 14), the second term of (3) can be expressed as

$$
H(X \mid Y)=\sum_{i=1}^{m} H\left(B_{i} \mid Y\right)
$$

where $B_{i}$ represents the $i^{\text {th }}$ binary channel associated to the $i^{t h}$ bit and $H\left(B_{i} \mid Y\right)$ is expressed as

$$
H\left(B_{i} \mid Y\right)=-\int_{y \in \mathbb{C}} p(y)\left(\int_{r \in \mathbb{R}^{2}} H\left(b_{i} \mid y ; r\right) P(r) \mathrm{d} r\right) \mathrm{d} y .
$$

The entropy $H\left(b_{i} \mid y ; r\right)$ is defined as

$$
H\left(b_{i} \mid y ; r\right)=-\sum_{s=0,1} P\left(b_{i}=s \mid y ; r\right) \log _{2} P\left(b_{i}=s \mid y ; r\right) .
$$

At this point, we are able to compute the mutual information of the Rayleigh fading channel for both the BICM and CM schemes. In the next section we propose to analyze the evolution of the mutual information as a function of the SSD rotation angle.

\section{EVAluATing MUtual INFORMATION TO OPTIMIZE SSD}

\section{A. Mutual information as a function of the rotation angle}

Figures 2 and 3 show the mutual information curves as a function of the SSD rotation angle for both the CM and the BICM schemes. Fig. 2 considers a 16-QAM with SNR values of 10,15 and $25 \mathrm{~dB}$. Fig. 3 considers a 256-QAM for SNR values of 15, 25 and $30 \mathrm{~dB}$. For the BICM scheme a Gray mapping is considered. Both figures show results for the flat fast Rayleigh fading channel with erasures $\left(p_{e}=0.1\right)$ and without erasure.

From these curves we can conclude that, in all cases, the CM scheme presents higher mutual information than the BICM. Also, rotation always provides gain for the $\mathrm{CM}$ scheme (unlike for the BICM, where rate loss may appear with rotation). Another point is that the variations on the mutual information are more significant in the erasure channel. Thus, the SSD can potentially provide more gains in the erasure channel.

In order to enhance the system performance with the SSD technique, we propose to select the rotation angle that maximizes the mutual information $I(X ; Y)$. For each $\mathrm{SNR}$, the optimal angle value can be expressed as

$$
\alpha_{o}(\mathrm{SNR})=\arg \max \left\{I(\alpha, \mathrm{SNR}), \alpha \in\left[0^{\circ}-90^{\circ}\right]\right\} .
$$
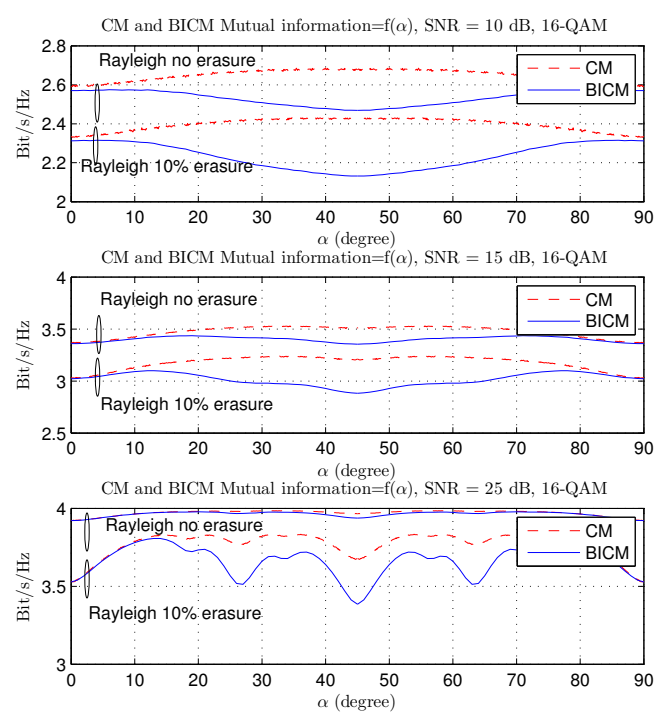

Fig. 2. Mutual information as a function of the rotation angle $\alpha$ for $\mathrm{CM}$ and BICM. SNR $=10,15$ and $25 \mathrm{~dB}$. Rayleigh fading channel and 16-QAM modulation.
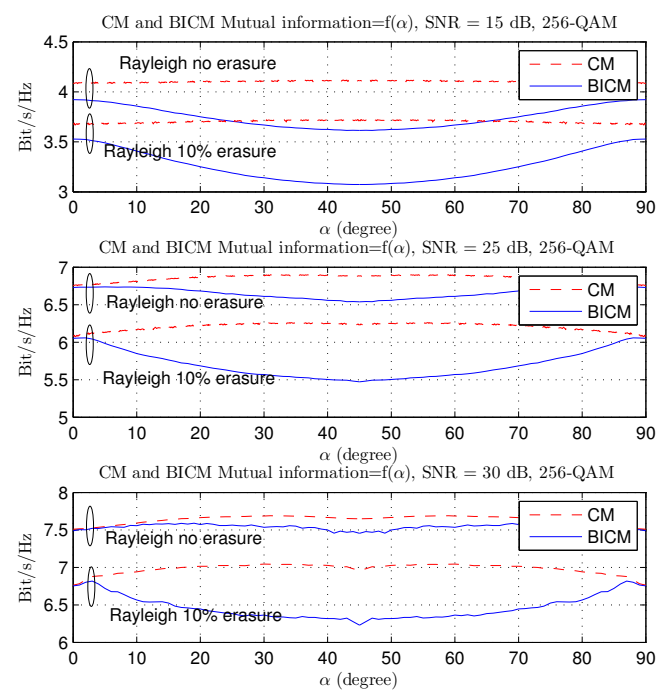

Fig. 3. Mutual information as a function of the rotation angle $\alpha$ for $\mathrm{CM}$ and BICM schemes. SNR $=15,25$ et $30 \mathrm{~dB}$. Rayleigh fading channel and 256-QAM modulation.

Note that this approach rationalizes the one proposed in [9], which implies a complex trade-off with several contradictory criteria.

Fig. 4 and Fig. 5 show the angles that maximize the mutual information for each SNR value (SNR $\in[0 \ldots 30]$ $\mathrm{dB}$ ) using respectively the 16-QAM and 256-QAM modulations. As in [8], for the BICM 16-QAM scheme, the optimal angle value for high SNR (i.e. greater that 25 $\mathrm{dB})$ is $\alpha_{o} \approx 31.7^{\circ}$. However for practical operating SNR values (between $18 \mathrm{~dB}$ and $25 \mathrm{~dB}$ ), $\alpha_{o} \approx 20^{\circ}$. If this angle value is considered for lower SNRs, a rate loss of about $0.03 \mathrm{bit} / \mathrm{s} / \mathrm{Hz}$ would be introduced at $\mathrm{SNR}=10$ $\mathrm{dB}$ with respect to the optimal angle $\alpha_{o}(10 \mathrm{~dB}) \approx 10^{\circ}$ (Fig. 2). For CM, the optimal angle value for high SNR is also $\alpha_{0}=31.7^{\circ}$. However, considering this angle also 


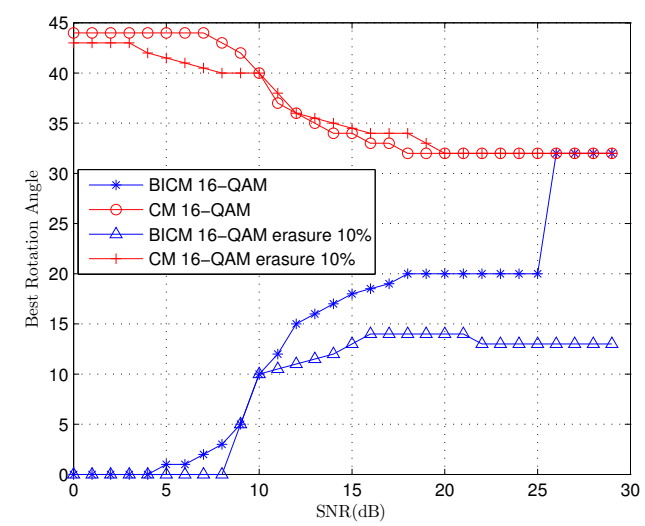

Fig. 4. Rotation angle that maximizes mutual information, as a function of the SNR, for the Rayleigh channel and Rayleigh channel with $10 \%$ erasures. 16-QAM modulation.

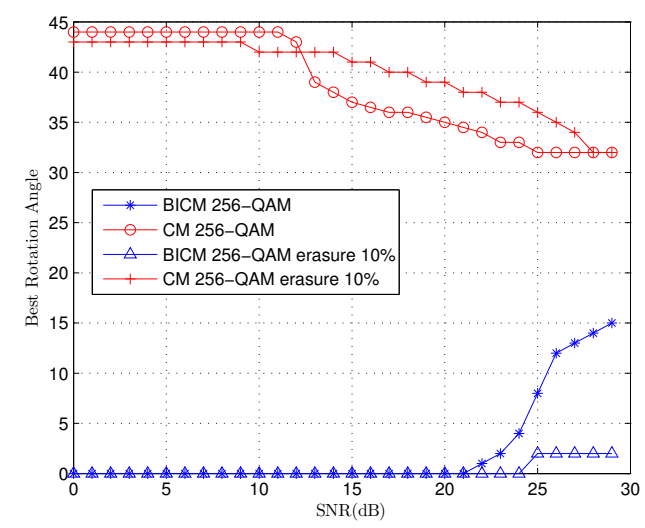

Fig. 5. Rotation angle that maximizes mutual information, as a function of the SNR, for the Rayleigh channel and Rayleigh channel with $10 \%$ erasures. 256-QAM modulation.

for lower SNRs barely affects performance (unlike for BICM): at $10 \mathrm{~dB}$, using $\alpha_{0}=31.7^{\circ}$ instead of the optimal angle introduces only a rate loss of $0.004 \mathrm{bit} / \mathrm{s} / \mathrm{Hz}$ for the 16-QAM (Fig. 2) and $0.003 \mathrm{bit} / \mathrm{s} / \mathrm{Hz}$ for the 256QAM. In other words, the choice of the same rotation angle for all SNR, introduces negligible rate loss for the CM scheme and a some tenths of rate loss for BICM. This fact was already observed in Figures 2 and 3, where the mutual information in the CM scheme is much less sensitive (about a factor of 10) to the angle variation than in BICM. Finally, with the erasure channel (Fig. 4 and 5), the optimal angles for the CM are closer to the angles with the channel with no erasure, giving a simple modulation scheme efficient for all channel conditions. This is not the case for the BICM modulation. In fact, for broadcasting applications where each receiver experiments a different level of SNR and erasure, the choice of the rotation angle is always a trade-off for BICM.

\section{B. Mutual information as a function of the SNR}

We now consider the mutual information curves as a function of the SNR for the selected angle values. Fig 6 considers the 16-QAM with rotation angles $\alpha_{o}=31.7^{\circ}$ for $\mathrm{CM}$ and $\alpha_{o}=16.8^{\circ}$ for BICM. This angle choice for the BICM is the one of the DVB-T2 standards (see Table I) and, from Fig. 4 as well, this angle choice is a good compromise for high and low SNRs. From these results, we observe that the $\mathrm{CM}$ with SSD outperforms the non-rotated scheme for all SNRs. This does not occur for BICM: at low SNR (up to approximately $12 \mathrm{~dB}$ for the 16-QAM), the rotation causes a mutual information loss compared to non-rotated BICM. This can also be observed in Figures 2 and 3, where the mutual information curve decreases with rotation. Therefore, the gains displayed by the rotated $\mathrm{CM}$ are more significant than for BICM.

The same curves are presented for the 256-QAM modulation in Fig. 7. The considered rotation angles are $\alpha_{o}=31.7^{\circ}$ for the $\mathrm{CM}$ and $\alpha=3.6^{\circ}$ for the BICM (see Table I). Using the BICM scheme, the SSD barely changes the mutual information performance and does not offer any significant advantage. However, for the CM scheme, a SNR gain greater than $1 \mathrm{~dB}$ can be observed for spectral efficiencies larger than $7 \mathrm{bit} / \mathrm{Hz} / \mathrm{s}$.

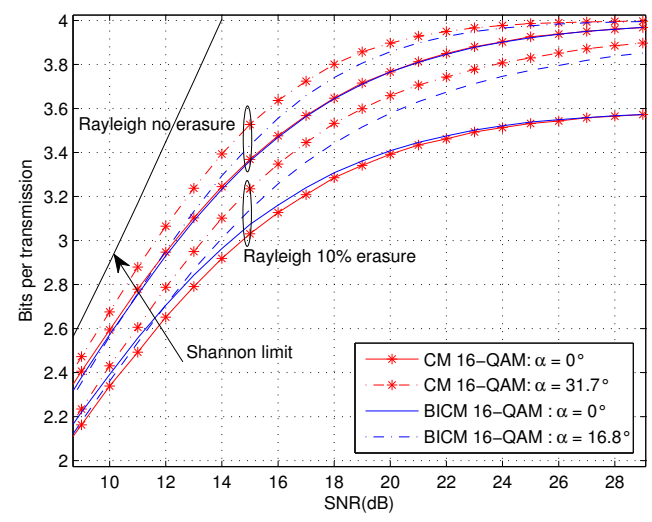

Fig. 6. $\mathrm{CM}$ and BICM mutual information curves for a 16-QAM modulation over fast flat fading Rayleigh channel (without erasures and with $10 \%$ erasures)

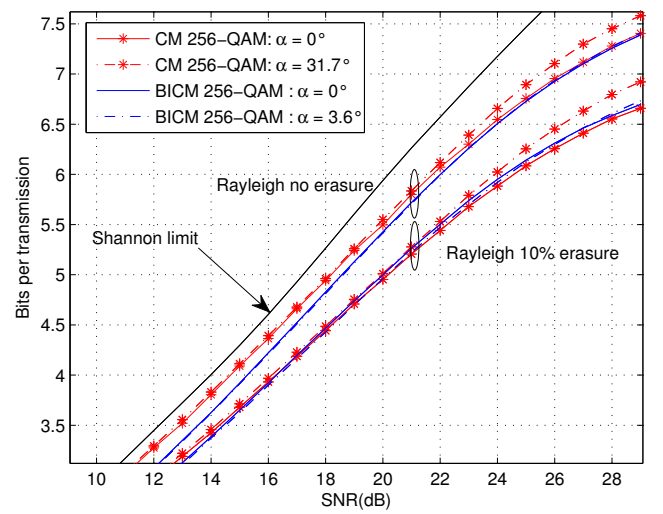

Fig. 7. CM and BICM mutual information curves for a 256-QAM modulation over fast flat fading Rayleigh channel (without erasures and with $10 \%$ erasures)

Over the erasure Rayleigh channel $\left(p_{e}=0.1\right)$, the gain introduced with the SSD is more significant than for the no-erasure case, for both 16- and 256-QAM and specially for high SNR values. 


\section{Simulation RESUlTS AND PERFORMANCE COMPARISON}

To verify the theoretical results, we ran Monte-Carlo simulations for the different scenarios considered in the previous sections. Because of lack of space, we only show results with the 256-QAM for two coding rates (but the same conclusions can be drawn from the simulation results with the 16-QAM). The BICM scheme considered is the one of the DVB-T2 standard, i.e., rotation angle of $3.6^{\circ}$, a specific interleaver pattern and an $N=64800$ binary irregular LDPC code. For the CM, two specific Non-Binary LDPC codes were designed for rates 3/4 and $9 / 10$. The length of these codes is 64800 bits or 8100 symbols defined over GF(256). These NB-LDPC codes are available at [10]. The decoding algorithm of the NBLDPC codes is the Extended Min-Sum (EMS) algorithm [11]. For the BICM, the LDPC decoder implements the Sum-Product algorithm followed by a $\mathrm{BCH}$ decoder as in [1]. We have used a maximum number of iterations equal to 50, same frame length and same channel conditions for both cases. Each point of the simulation curves represents the transmission of data until 100 erroneous frames have been encountered. For the EMS decoding, we have performed simulations with $n_{m}=50$, i.e. length of the exchanged messages between check and variable nodes [11].

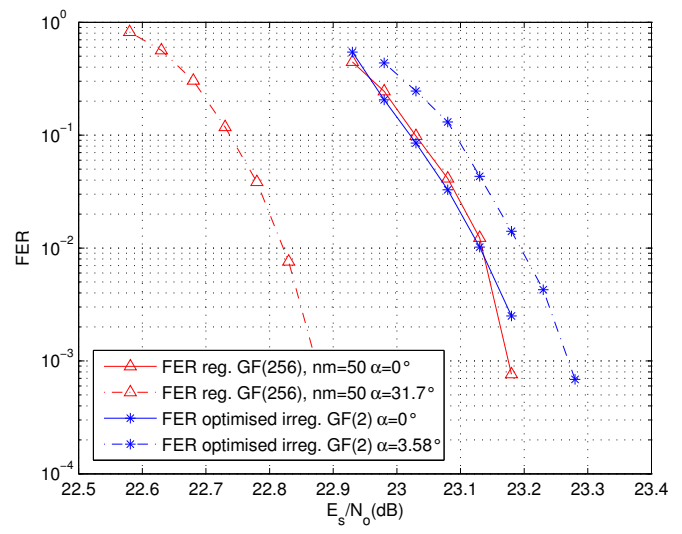

Fig. 8. FER simulation for 3/4-rate BICM-GF(2) and CM-GF(256) schemes over the the fast flat Rayleigh fading channel, with and without Rotated Constellation

Fig. 8 presents results for a code rate of $3 / 4$. As already observed in Figure 3, the SSD technique introduces performance loss in the BICM scheme for spectral efficiencies lower than $7 \mathrm{bit} / \mathrm{Hz} / \mathrm{s}$. However, as announced by the theoretical study, a gain of about $0.3 \mathrm{~dB}$ is provided by the SSD technique for CM.

For higher spectral efficiencies (9/10-rate in Fig. 9), SSD introduces gain for both the BICM and CM schemes, as certified by a zoom on the right upper zone of Fig. 7. This gain is more significant in the $\mathrm{CM}$ scheme $(1.3 \mathrm{~dB}$ at FER $=10^{-2}$ compared to about $0.15 \mathrm{~dB}$ for BICM). Note that these performance results are consistent with the analysis of Fig. 3.

\section{CONCLUSiON}

In this paper we considered the application of the signal space diversity technique to high spectral efficiency com-

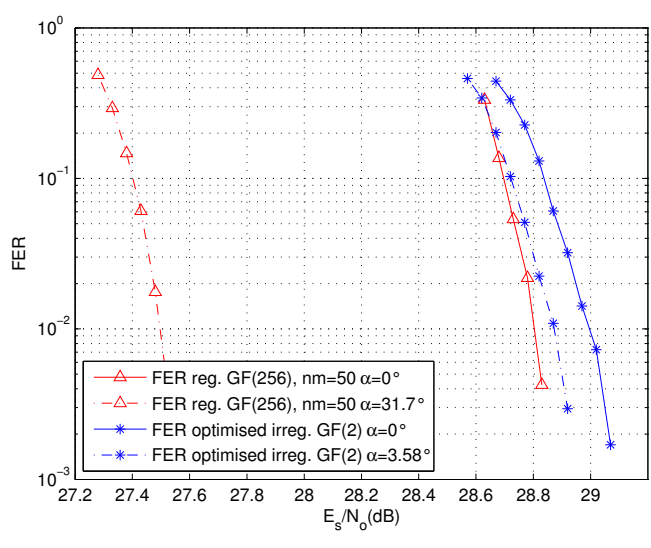

Fig. 9. FER simulation for 9/10-rate BICM-GF(2) and CM-GF(256) schemes over the the fast flat Rayleigh fading channel, with and without Rotated Constellation

munications in the context of next generation broadcasting systems. We proposed to determine the optimal rotation angle as the one that maximizes the mutual information for practical signal-to-noise ratio values. We showed that non-binary coded modulations are more robust than bitinterleaved coded modulations and take better advantage of the signal space diversity technique. The solutions that we proposed can lead to significant performance gains for next generation broadcasting systems.

\section{REFERENCES}

[1] "Digital Video Broadcasting (DVB); frame structure, channel coding and modulation for a second generation digital terrestrial television broadcasting system (DVB-T2)," in ETSI EN 302755 , 2008.

[2] D. Gomez-Barquero, C. Douillard, P. Moss, and V. Mignone, "DVB-NGH: The next generation of digital broadcast services to handheld devices," IEEE Transactions on Broadcasting, vol. 60, no. 2, pp. 246-257, June 2014.

[3] J. Boutros and E. Viterbo, "Signal space diversity: a power and bandwidth-efficient diversity technique for the Rayleigh fading channel," IEEE Transactions on Information Theory,, vol. 44, no. 4, pp. 1453-1467, Jul. 1998.

[4] E. Zehavi, "8-PSK trellis codes on Rayleigh channel," in IEEE Military Communications Conference (MILCOM), Oct. 1989, pp. 536-540 vol.2

[5] G. Caire, G. Taricco, and E. Biglieri, "Bit-interleaved coded modulation," IEEE Transactions on Information Theory, vol. 44, no. 3, pp. 927-946, May 1998.

[6] Z. Ma, Z. Shi, C. Zhou, and Z. Zhang, "Design of signal space diversity based on non-binary LDPC code," in International Conference on Communications, Circuits and Systems, May 2008, pp. 31-34.

[7] W. Zhou and L. Zou, "Optimized rotations and labeling for nonbinary LDPC coded modulation with signal space diversity," in IEEE International Symposium on Broadband Multimedia Systems and Broadcasting (BMSB), May 2009, pp. 1-4.

[8] C. Abdel Nour and C. Douillard, "Improving BICM performance of QAM constellations for broadcasting applications," in 5th International Symposium on Turbo Codes and Related Topics, Sep. 2008 , pp. $55-60$.

[9] — , "Rotated QAM constellations to improve BICM performance for DVB-T2," in IEEE 10th International Symposium on Spread Spectrum Techniques and Applications, Aug. 2008, pp. 354 - 359.

[10] C. Marchand and E. Boutillon. (2015, Jul.) Lab-STICC website on Non-Binary LDPC. [Online]. Available: http://www-labsticc. univ-ubs.fr/nb_ldpc/

[11] D. Declercq and M. Fossorier, "Decoding algorithms for nonbinary LDPC codes over GF(q)," IEEE Transactions on Communications, vol. 55, no. 4, pp. 633 - 643, Apr. 2007. 